\author{
Edwin A. M. Gale
}

\title{
Your journal and mine
}

Published online: 24 December 2004

(C) Springer-Verlag 2004

The American Diabetes Association was founded in 1940, at a time when there were thought to be nearly one million people with diabetes in the United States. The founders were concerned about the rising incidence of the condition, attributed to increasing obesity, and they pointed out that diabetes carried the same mortality as tuberculosis but affected many more people. The European Association for the Study of Diabetes was established in 1965, at a time when no one knew how many Europeans were affected. Each Association came out with its own journal; Diabetes (published under that name from 1952) was the obvious title for the Americans, while our own predecessors opted for the quaint and unpronounceable Diabetologia. Those who wish to speak the name should note that all seven vowels are enunciated, that the third syllable can be pronounced either "bet" or "beat" and that the emphasis can be on the third, fourth, fifth or sixth vowels according to preference. It's up to you. So why did we choose such an unappealing title? Let me guess that the question as to whether the title should be in French, German or English was resolved in favour of a language that nobody speaks -a quintessentially European solution. The original journal was indeed published in all three languages. Even now we retain our distinctive flavour, with authentic British English spelling, as against the dialect in use elsewhere.

The number of people with diabetes has risen rapidly since our two Associations were launched, but not nearly as fast as the number of scientific articles about their condition. In 1978 the ADA responded by launching Diabetes Care, a second and more clinically orientated journal. This continues to go from strength to strength and to climb the impact factor ratings in the capable hands of

E. A. M. Gale $(\bowtie)$

Medical School Unit, Southmead Hospital, University of Bristol,

Southmead Road,

Bristol, BS10 5NB, UK

e-mail: diabetologia-j@bristol.ac.uk

Tel.: +44-117-9595338

Fax: +44-117-9595352
Mayer Davidson, the current Editor. The founding Editors of Diabetologia saw their task as building bridges between basic science and clinical care, and our journal continues to publish articles on diabetes in all its aspects. Should we still do so? Or should we accept that the gap between basic science and clinical care is expanding so rapidly that few readers will have the interest or breadth of understanding to examine more than a small fraction of the papers we publish? My own view is that bridges are needed, now more than ever, and that we should try and build better ones. Your "new look" journal is a step towards this. We now have a pictorial cover, with a theme for each year. Year One will celebrate some of the Past Masters who helped to create the tradition of enquiry and care that we inherit. As you will see from the details provided by Viktor Jörgens, we chose Apollinaire Bouchardat for the first cover because he dreamed of a united Europe 140 years ago, and proposed that diet and exercise should form the basis of diabetes therapy. Practical implementation of both aspirations remains a challenge, but he showed us the way to go.

The change goes deeper than the cover. We have no wish to preside over a mausoleum of decently interred scientific articles: our aim is a journal that people will want to read. This means a more even balance between clinical and experimental diabetes, more commentary and debate, and good quality reviews that are accessible to a wide readership. In the last analysis, however, a journal is only as good as the original manuscripts it attracts, and here we need to try harder. To begin with, we have - at last - changed to electronic submission. This could have the effect of excluding items by your own Editor, who has never yet succeeded in submitting an electronic manuscript without assistance, but those with teenage children should have no problems. We are increasing our number of pages (still with no page charges), and are working closely with our publishers to streamline the way we process manuscripts, with the aim of on-line publication very soon after acceptance. We have launched our own website (http://www.diabetologia-journal.org), hosted by the EASD and linked to the journal contents on the 
Springer site. Not least, we have changed the editorial structure of the journal, with much greater responsibility (and more work!) devolved to our Associate Editors.

All this has forced us to think more carefully about the standards we expect from manuscripts in any given area, and how to achieve a more uniform quality for the journal. We acknowledge the immense debt owed to our referees, and it is still a matter of astonishment and awe that so many people are prepared to donate their time to evaluation of our papers, given that their only thanks for a job well done is to receive yet another manuscript. We cannot reward our referees, but we can give them more recognition. For this reason, we now publish a list of key referees - those who have provided three or more helpful reports in timely fashion in the course of a year. We use these people because they are good at what they do. Go to the back of this issue and look for your name. Is it there?

Those with an interest in history will know that most journals were launched by learned societies in order to promote their chosen area of knowledge, and to define standards within this area. Acceptance of a new memberor a new article for the journal-signified that a certain standard had been met; our anonymous peer-review system is inherited from the methods used by exclusive nineteenth-century clubs to solve this particular problem. Journals are therefore defined by what they exclude. This has a sad and ludicrous consequence; ask colleagues how they are getting on, and they will reply with the news that they have a paper coming out in Nature or some other well-known journal; the content will be considered of secondary importance. Publications have become an academic body count, a way of keeping score rather than a means of communication.

A novice Editor is soon confronted by the pathology of a system in which academic careers depend upon the number of publications in high-status journals. This is not without its lighter aspects: some groups appear to employ a secretary whose task is simply to post rejected articles on to the next journal in line. How else do we explain three recent papers with covering letters requesting consideration for publication in another journal? Far from reading or responding to the advice of referees, such groups may not even bother to correct the typographical errors that have been drawn to their attention. This tends to get noticed by referees, especially those who spent an hour or two trying to help the authors last time round. And let us award points for sheer cheek to the authors who submitted the same manuscript on the same day to an American journal and to ourselves. If there were a Darwin Award for suicidal publishing behaviour, these would surely be among the winners. Others are more subtle, and aim to evade detection by the tactic of submitting closely related articles in parallel to different journals without citing the cloned version. No fewer than five groups of authors have asked me to believe they simply forgot to mention the existence of a manuscript concerning the same study that had just been submitted or published. Not all cheats are detected at this stage, and I invite readers to alert us to papers we publish that fail to cite a parallel report by the same authors in another journal. Those who do so will get a nice bottle of wine from myself, and the authors will receive an Amnesia Award in the form of a published Erratum.

A journal must stand up for good science, and this knows no boundaries. We welcome papers from around the world, and judge our progress by the proportion from other continents. We relish the diversity we receive, particularly when it comes to approaches to clinical care. We appreciate that diabetes has become a matter of urgent public concern, and that the way in which a society looks after people who suffer from a chronic disease such as this reflects the culture and values of that society. The United States leads us all in science and health technology, but not when it comes to caring for the health of its own people. With the most expensive health care system in the world, it ranks 29th in terms of healthy life expectancy, and $15.6 \%$ of Americans, including many of the elderly and vulnerable, lack any health insurance. The approach to health care favoured by most countries in Europe and elsewhere is better suited to confront the wider challenge of diabetes. This will be part of our message. And finally, the journal will celebrate the progress of the EASD, which recently hosted the diabetes meeting with the largest number of registered participants on record. Guided by a series of able Presidents, it now funds its own research and speaks up for the diabetes research community in Europe and around the world. This is your journal, and we are on the move. 